\title{
COMBATE QUÍMICO DE THRIPS TABACI (THYSANOPTERA: THRIPIDAE) EN EL CULTIVO DE CEBOLLA, EN MORELOS, MÉXICO
}

\section{CHEMICAL CONTROL OF THRIPS TABACI (THYSANOPTERA: THRIPIDAE) IN ONION CROP OF MORELOS, MEXICO}

\section{Cid AgUILAR CARPIO, ${ }^{1, *}$ Arturo GONZÁleZ RENDÓN, ${ }^{2}$ Adriana PÉREZ RAMÍreZ, ${ }^{3}$ SERgio Gavino RAMÍREZ ROJAS ${ }^{4}$ Y VICENTE EMILIo CARAPIA RUIZ ${ }^{2}$}

\author{
${ }^{1}$ Universidad Autónoma del Estado de Morelos. Facultad de Ciencias Agropecuarias. Av. Universidad No. 1001, \\ Col Chamilpa, Cuernavaca, Morelos, México. C.P. 62209. \\ ${ }^{2}$ Escuela de Estudios Superiores de Xalostoc. Av. Nicolás Bravo s/n, Parque Industrial Cuautla, Xalostoc, C.P. 62717. \\ ${ }^{3}$ IDAGRO S. de R. L. de C. V. Carretera Yautepec-Tlayacapan S/N. Col. Puente Pantitlan. Tlayacapan, Mor. \\ C. P. 62545. \\ ${ }^{4}$ INIFAP- Campo Experimental de Zacatepec. Km. 0.5 Carretera Zacatepec-Galeana, Col. Centro C.P. 62780, \\ Zacatepec, Morelos. \\ * Autor para correspondencia: <aguilar.cid@colpos.mx>
}

Recibido: 02/03/2016; aceptado: 10/01/2017

Editor responsable: Jesús Romero Nápoles

Aguilar C., C., González R., A., Pérez R., A., Ramírez R., S. G. y Carapia R., V. E. (2017). Combate químico de Thrips tabaci (Thysanoptera: Thripidae) en el cultivo de cebolla en Morelos, México. Acta Zoológica Mexicana (n.s.), 33(1), 39-44.

RESUMEN. El cultivo de cebolla presenta altas poblaciones del trips (Thrips tabaci Lindeman), lo que ocasiona marchitez prematura, retarda el desarrollo de la hoja y distorsiona los brotes vegetativos. Los agricultores han usado diferentes plaguicidas para el manejo del trips, sin tener en cuenta la rotación de grupos químicos y dosis adecuadas, lo que ocasiona contaminación ambiental por el uso excesivo de agroquímicos. Por lo que el objetivo de este estudio, fue evaluar seis grupos de plaguicidas para el manejo de trips, los cuales fueron rotados durante el ciclo del cultivo, así como también se identificó la especie de trips presente en el cultivo de la cebolla. El estudio inició en octubre de 2013, en un cultivo de cebolla, ubicada en Ayala, Morelos. Cada tratamiento consistió en la aplicación de los siguientes insecticidas: T1: Testigo (sin producto); T2: Movento, Velcefate, Muralla, Tracer; T3: Regent, Muralla, Movento, Seizer+ Agromectin; T4: Tracer, Movento, Curacron, Muralla; T5: Actigard, Tracer, Colt, Agromectin; T6: Evisect-s, Curacron, Tracer, Calypso. Para determinar el control de los insecticidas, se contabilizó el número de ninfas y adultos de trips. Con los datos del número de ninfas y adultos de trips en los diferentes tratamientos, se realizó un análisis de varianza y separación de medias con la prueba Tukey ( $\alpha 0.05)$. Para determinar las especies de trips presentes, se tomó una muestra en las hojas de las plantas de cebolla. Los insecticidas que lograron un control de las ninfas de trips en el cultivo de cebolla, fueron el Tracer, Acefate, Curacron y la combinación de Seizer+ Agromectin. Los insecticidas Tracer y Muralla, fueron los que mantuvieron las poblaciones más bajas de adultos de trips en el cultivo de cebolla. La especie de trips predominante en el cultivo de cebolla durante el estudio fue Thrips tabaci L.

Palabras clave: Trips, control químico, cebolla.
Aguilar C., C., González R., A., Pérez R., A., Ramírez R., S. G., \& Carapia R., V. E. (2017). Chemical control of Thrips tabaci (Thysanoptera: Thripidae) in onion crop of Morelos, Mexico. Acta Zoológica Mexicana (n.s.), 33(1), 39-44.

ABSTRACT. The onion crop has high populations of thrips (Thrips tabaci Lindeman), which causes premature wilting, retard leaf development and distorts vegetative buds. Farmers have been used different pesticides for managing trips, regardless of the rotation of chemical groups and inadequate doses, causing environmental pollution and overuse of agrochemicals. The objective of this study was to evaluate six groups of pesticides for the management of thrips, which were rotated during the growing season and the thrips species present in an onion crop also was identified. The study began in October 2013 in an onion crop, located in Ayala, Morelos. Each treatment consisted of the application of the following insecticides: T1: Control (no products); T2: Movento, Velcefate, Muralla, Tracer; T3: Regent, Muralla, Movento, Seizer+ Agromectin; T4 Tracer, Movento, Curacron, Muralla; T5 Actigard, Tracer, Colt, Agromectin; T6 Evisect-s, Curacron, Tracer, Calypso. To determine control of insecticides, the number of nymphs and adult thrips were counted. Analysis of variance and mean separation test with Tukey $(\alpha 0.05)$ was performed with data on the number of nymphs and adult thrips. To determine the species of thrips present, a sample was taken from the leaves of onion plants. Insecticides that achieve control of nymphs of thrips in onion crop were the Tracer, Acephate, Curacron and combining Seizer + Agromectin. The Tracer and Muralla insecticides were those who maintained the lowest adult thrips in onion growing populations. The predominant species of thrips in onion crop during the study was Thrips tabaci L.

Key Words: Thrips, chemistry control, onion. 


\section{INTRODUCCIÓN}

La cebolla (Allium cepa L.) se produce en varios países del mundo y México se encuentra dentro de los 12 principales países productores (FAO 2010). A nivel nacional y durante el año agrícola 2013, fue la quinta hortaliza más importante con una superficie sembrada de 43,561 ha $y$ una producción de 1,270,059 t, figurando dentro de los principales estados productores Baja California, Guanajuato, Chihuahua, Puebla y Zacatecas. Mientras que el estado de Morelos se ubica en la octava posición, con una superficie de 3,292 ha y una producción de 91,242 t (SIAP 2013).

El cultivo de cebolla presenta problemas fitosanitarios ocasionados por distintas plagas (García \& González 2010), entre las que destacan: el gusano soldado (Spodoptera exigua (Hübner)), el minador de la hoja (Liromyza spp.) y la gallina ciega (Phyllophaga spp. y Cyclocephala spp.). Sin duda la plaga que más destaca por su capacidad invasiva, amplia distribución y polifagia es el trips de la cebolla: Thrips tabaci Lindeman (Thysanoptera: Thripidae) (Reveles-Hernández et al. 2014). Este insecto polífago, se hospeda en diferentes cultivos hortícolas como tomate de cáscara, jitomate, papa, ajo, cebolla, algodonero, tabaco, entre otros cultivos y maleza (Ramírez et al. 2010). Cuando las poblaciones son numerosas pueden ocasionar marchitez prematura, retardar el desarrollo de la hoja y distorsionar los brotes (Alston 2008). En los estados de Zacatecas y Morelos, se detectó una enfermedad en los cultivos de cebolla y ajo durante el año 2011, causada por la infección del tospovirus Iris yellow spot virus (IYSV), misma que se encuentra asociada a la presencia de Thrips tabaci (Riley et al. 2011, Velázquez \& Reveles 2011). Asimismo, en el estado de Morelos y durante el ciclo agrícola otoño-invierno 2012-2013, se presentaron altas poblaciones de trips y síntomas característicos del IYSV en los cultivos de cebolla ubicados en los municipios de Axochiapan, Tepalcingo y Xochitepec, los cuales fueron asociados con pérdidas desde 50 hasta $100 \%$ en el rendimiento del cultivo de cebolla. (Osuna-Canizalez \& Ramírez-Rojas 2013). Al respecto, se han realizado estudios para el control químico de trips en diferentes partes del mundo como el de Al Mazraáwi (2007), Workman \& Martin (2002) y Turini (2011), donde encontraron que el mejor programa para el manejo del trips fue la rotacion de los siguientes productos: spinetoram, spirotetramat, acetamiprid y metomilo; sin embargo, los agricultores han usado diferentes plaguicidas para el manejo del trips, sin tener en cuenta la rotación de grupos químicos y dosis adecuadas, lo que ha ocasionado contaminación al ambiental por el uso excesivo de agroquímicos y en consecuencia un mayor costo de producción. Por lo que el objetivo de este estudio, fue evaluar seis grupos de rotación de insecticidas para el manejo de trips, así como identificar la especie de trips presente en el cultivo de la cebolla, en Ayala, Morelos, México.

\section{MATERIALES Y MÉTODOS}

El estudio inició el 28 de octubre de 2013, en un cultivo de cebolla de la variedad Cirrus en Huitzililla, municipio de Ayala, Morelos. A los 17 días después del trasplante del cebollín y hasta cinco días antes de la cosecha se hicieron las pruebas con los insecticidas; se aplicaron seis tratamientos, los cuales fueron rotando los distintos grupos de insecticidas utilizados. En este estudio, cada tratamiento consistió de la aplicación de cuatro insecticidas (algunos de éstos se formaron a base de mezclas) de diferente grupo químico, cada insecticida se aplicó durante tres semanas ( \pm 1 semana). La primera aplicación de los insecticidas se inició a los 18 días después de la siembra y una vez que la planta había emergido, posteriormente se realizaron 10 aplicaciones más con intervalos de 7 días entre cada una. La aplicación se realizó vía foliar con un aspersor manual de $15 \mathrm{~L}$ equipado con una boquilla de cono y calibrado para aplicar un gasto de $400 \mathrm{~L}$ de agua por hectárea. Los tratamientos e insecticidas utilizados en el experimento se indican en el Cuadro 1.

El estudio se estableció en un diseño experimental de bloques completamente al azar con seis tratamientos y cuatro repeticiones. La unidad experimental se formó por cinco surcos de $4 \mathrm{~m}$ de largo y $0.55 \mathrm{~m}$ entre surcos, obteniendo un área de $11 \mathrm{~m}^{2}$ por unidad experimental. La parcela útil fueron los tres surcos centrales, exceptuando $0.5 \mathrm{~m}$ en cada extremo de los surcos.

Para determinar el control de los tratamientos, se realizó un muestreo previo al inicio del estudio y otro a los seis días después de la primera aplicación. Después de la segunda aplicación, se muestreó al día siguiente de cada aplicación y a los seis días después de la misma, realizando un total de 22 muestreos durante todo el estudio. En cada muestreo se contabilizó el número de ninfas y adultos vivos de trips presentes en las tres últimas hojas emergidas de cada planta, tomando una muestra de 10 plantas al azar dentro de la parcela útil por repetición, lo que dio un total de 40 plantas por tratamiento. Debido a la variabilidad de los datos del número de ninfas y adultos 
Cuadro 1. Tratamientos e insecticidas utilizados en el cultivo de cebolla.

\begin{tabular}{|c|c|c|c|c|}
\hline Tratamiento & Productos* & & & \\
\hline 1 & Testigo (sin producto) & & & \\
\hline 2 & $\begin{array}{l}\text { Movento (Spirotretamat) } \\
400 \mathrm{~mL} \mathrm{ha}^{-1}\end{array}$ & $\begin{array}{l}\text { Velcefate (Acefate) } \\
800 \mathrm{~g} \mathrm{ha}^{-1}\end{array}$ & $\begin{array}{l}\text { Muralla (Imidacloprid }+ \\
\text { Betacyflutrin) } \\
400 \mathrm{~mL} \mathrm{ha}^{-1}\end{array}$ & $\begin{array}{l}\text { Tracer (Spinosad) } \\
100 \mathrm{~mL} \mathrm{ha}^{-1}\end{array}$ \\
\hline 3 & $\begin{array}{l}\text { Regent (Fipronil) } \\
150 \mathrm{~mL} \mathrm{ha}^{-1}\end{array}$ & $\begin{array}{l}\text { Muralla (Imidacloprid + } \\
\text { Betacyflutrin) } \\
400 \mathrm{~mL} \mathrm{ha}^{-1}\end{array}$ & $\begin{array}{l}\text { Movento (Spirotretamat) } \\
400 \mathrm{~mL} \mathrm{ha}^{-1}\end{array}$ & $\begin{array}{l}\text { Seizer (Bifentrina) } 0.6 \mathrm{~L} \mathrm{ha}^{-1} \\
+ \text { Agromectin (Abamectina) } \\
1 \mathrm{~L} \mathrm{ha}^{-1}\end{array}$ \\
\hline 4 & $\begin{array}{l}\text { Tracer (Spinosad) } \\
100 \mathrm{~mL} \mathrm{ha}^{-1}\end{array}$ & $\begin{array}{l}\text { Movento (Spirotretamat) } \\
400 \mathrm{~mL} \mathrm{ha}^{-1}\end{array}$ & $\begin{array}{l}\text { Curacron(Propenofos) } \\
400 \mathrm{~mL} \mathrm{ha}^{-1}\end{array}$ & $\begin{array}{l}\text { Muralla (Imidacloprid }+ \\
\text { Betacyflutrin) } 400 \mathrm{~mL} \mathrm{ha}^{-1}\end{array}$ \\
\hline 5 & $\begin{array}{l}\text { Actigard (Acibenzolar S metil) } \\
30 \mathrm{~g} \mathrm{ha}^{-1}\end{array}$ & $\begin{array}{l}\text { Tracer (Spinosad) } \\
100 \mathrm{~mL} \mathrm{ha}\end{array}$ & $\begin{array}{l}\text { Colt (Imidacloprid) } \\
350 \mathrm{~L} \mathrm{ha}^{-1}\end{array}$ & $\begin{array}{l}\text { Agromectin (Abamectina) } \\
1 \mathrm{~L} \mathrm{ha}^{-1}\end{array}$ \\
\hline 6 & $\begin{array}{l}\text { Evisect-s (Tiocyclam - hidrogenoxalato) } \\
600 \mathrm{~g} \mathrm{ha}^{-1}\end{array}$ & $\begin{array}{l}\text { Curacron (Propenofos) } \\
400 \mathrm{~mL} \mathrm{ha}^{-1}\end{array}$ & $\begin{array}{l}\text { Tracer (Spinosad) } \\
100 \mathrm{~mL} \mathrm{ha}^{-1}\end{array}$ & $\begin{array}{l}\text { Calypso (Thiacloprid) } \\
0.2 \mathrm{~L} \mathrm{ha}^{-1}\end{array}$ \\
\hline
\end{tabular}

*Grupo toxicológico de cada insecticida, Movento: Ácido tetrámico; Velcefate: Organofosforado; Tracer: Spinosine; Calypso: Cloronicotinilos (Cianamida); Agromectin: Lactona macrocíclica glucosidada; Colt: Cloronicotinilos; Regent: Fenilpirazoles; Curacron: Organofosforado; Evisect-s: Análogo de la nereistoxina; Actigard: Benzotiadiazol; Muralla: Cloronicotinilos + Piretroide; Seizer: Piretroide + Lactona macrocíclica glucosidada.

de trips, se realizó una transformación de los datos originales con la formula $\sqrt{ }(\mathrm{x}+1)$ para posteriormente realizar el análisis de varianza con el programa estadístico de SAS Versión 9.0 (SAS, 2003) y la separación de medias con la prueba Tukey $(\alpha=0.05)$.

Para determinar las especies de trips presentes en el cultivo de cebolla, se tomaron muestras de trips en las últimas hojas formadas por las plantas en cada unidad experimental; las colectas se realizaron cada siete días, a partir del muestreo inicial y cinco días antes de la cosecha. Para recolectar los insectos, se utilizaron hisopos de algodón previamente humedecidos en alcohol al 70\% y posteriormente se colocaron en tubos Eppendorf de $2 \mathrm{~mL}$ con alcohol al 70\%, para conservarlos antes de montarlos e identificarlos, las muestras se llevaron al laboratorio de Entomología y Acarología de la Escuela de Estudios Superiores de Xalostoc de la Universidad Autónoma del Estado de Morelos, los caracteres morfológicos se observaron mediante un microscopio compuesto, utilizando la técnica de montaje en portaobjetos descrita por Johansen \& Mójica (1997); para la determinación de las especies de trips se utilizaron las claves taxonómicas de Mound \& Marullo (1996), Mound \& Kibby (1998) y Palmer et al. (1989).

\section{RESULTADOS Y DISCUSIÓN}

Identificación de trips. En total se realizaron 288 montajes de especímenes, de éstos se determinó la presencia de dos especies, Thrips tabaci y Frankliniella occidentalis Pergande; del total de las colectas se logró obtener la proporción der éstas, 1,415 individuos de T. tabaci y 41 de $F$. occidentalis.

Dinámica poblacional del trips en el cultivo de cebolla. Al inicio del estudio, cuando el cultivo de cebolla se encontraba en etapa de formación de hojas, las poblaciones de T. tabaci eran muy bajas, oscilando los promedios de ninfas entre 1.37 a 3.2 ninfas vivas por planta; mientras que los adultos con un promedio de 0.66 a 1.4 individuos vivos por planta (Figura 1). El máximo poblacional en ninfas se registró al inicio de la formación del bulbo, esto en el mes de diciembre. Al respecto Lewis (1973), mencionó que el aumento en la población de trips estuvo relacionado con el hábito de alimentación de este insecto, situación que ocurre en la formación del bulbo, donde la planta tiene más hojas. Por otro lado, los adultos oscilaron en promedios de 3 a 21 individuos vivos por planta. Para los muestreos posteriores la población de ninfas fue disminuyendo considerablemente, a finales del mes de diciembre e inicio de enero cuando descendió la temperatura no se encontraron ninfas sólo adultos; esto probablemente pudo deberse a que la temperatura bajó durante estos muestreos; al respecto, Samikura (1937) registró que los machos de T. tabaci perecen durante el invierno. Nuestros resultados se relacionan con lo mencionado por Burnstone (2009), quien en un muestreo realizado durante la época de invierno, las ninfas presentaron valores de cero y los adultos oscilaron entre 65 a 120. En la Figura 1 se puede observar las condiciones térmicas en las que 


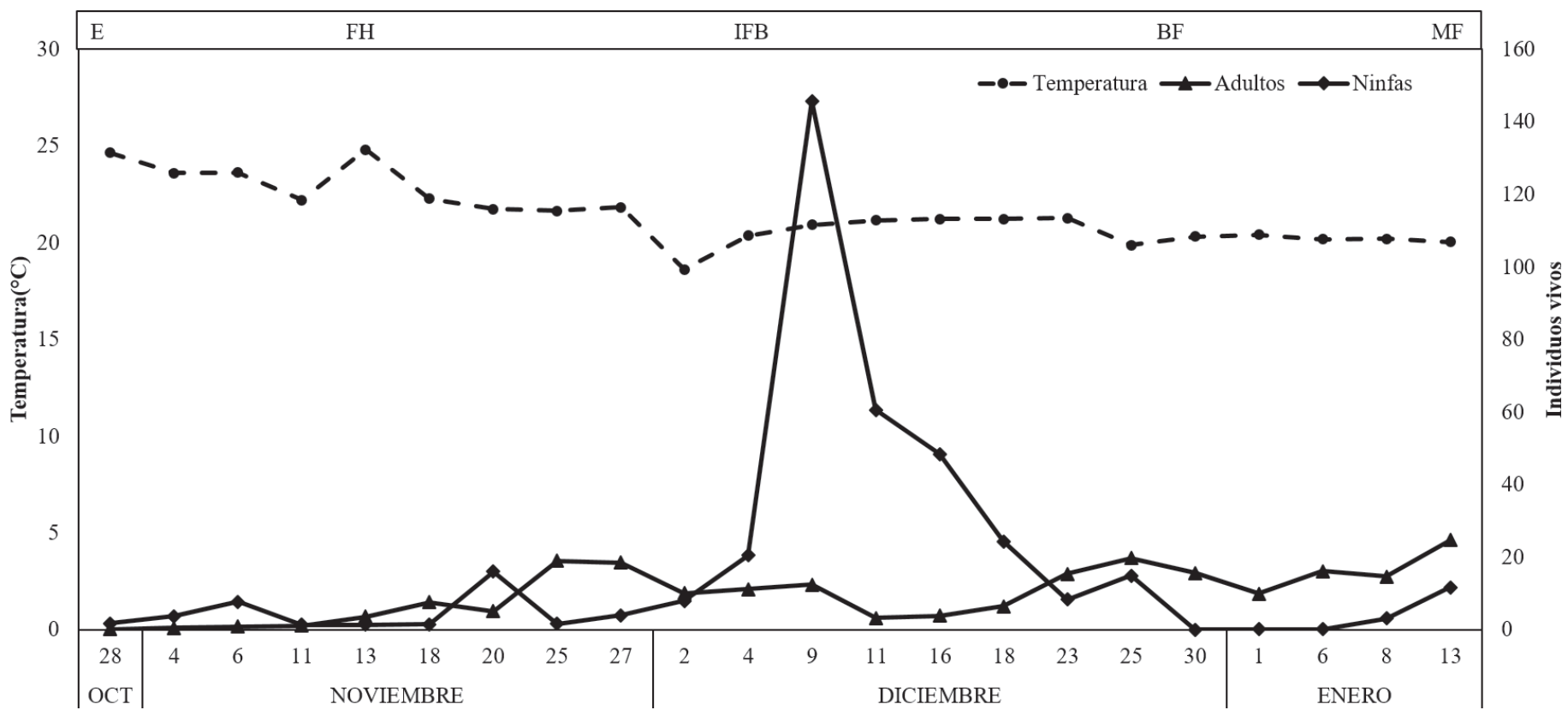

Figura 1. Dinámica poblacional de adultos y ninfas de trips en el testigo, en Huitzililla municipio de Ayala, Morelos 2013. E = Emergencia, FH = Formación de hojas, IFB = Inicio de formación de bulbo, BF = Bulbo formado, MF = Madurez fisiológica.

se desarrolló nuestro cultivo, que fue de 16.92 a 26.50 ${ }^{\circ} \mathrm{C}$, temperaturas óptimas para la propagación del trips, ya que las temperaturas que necesita para completar su ciclo de vida oscila de acuerdo a la temperatura, por ejemplo el ciclo podría tardar 37 días a $15{ }^{\circ} \mathrm{C} ; 21$ días a $20{ }^{\circ} \mathrm{C}$ y sólo 12 días a $30^{\circ} \mathrm{C}$ (García 2003).

Efecto de los grupos de rotación de insecticidas 1, 2, 3 y 4 sobre la población de ninfas de $T$. tabaci. En el primer grupo de rotación de insecticidas no se observó un efecto positivo, justo como lo mostró la prueba de comparación de medias Tukey que agrupó a todos los tratamien- tos con la misma letra (Cuadro 2). Este primer resultado se pudo deber a las bajas poblaciones que se presentaron en la primera aplicación. En cuanto al segundo grupo de insecticidas, el mejor tratamiento fue Acefate, debido a que controló el mayor número de ninfas en comparación con los demás insecticidas (Liñan \& Liñan, 2012); en tanto que el testigo presentó un incremento en las poblaciones de ninfas en relación al muestreo previo. Al parecer la efectividad del Acefate se debió a que es un insecticida de ingestión y contacto, contrario a los demás insecticidas que actúan de forma sistémica (Liñan \& Liñan, 2012).

Cuadro 2. Promedio de los tratamientos aplicados para el control de ninfas de trips.

\begin{tabular}{|c|c|c|c|c|c|c|c|c|}
\hline \multirow{2}{*}{$\begin{array}{c}\text { Tratamiento } \\
\mathrm{T} 1\end{array}$} & \multicolumn{2}{|c|}{ Grupo de rotación 1} & \multicolumn{2}{|c|}{ Grupo de rotación 2} & \multicolumn{2}{|c|}{ Grupo de rotación 3} & \multicolumn{2}{|c|}{ Grupo de rotación 4} \\
\hline & Testigo & $3.22 \mathrm{a}$ & Testigo & $8.65 \mathrm{a}$ & Testigo & $50.43 \mathrm{a}$ & Testigo & $3.31 \mathrm{a}$ \\
\hline $\mathrm{T} 2$ & Movento & $3.24 \mathrm{a}$ & Acefate & $0.25 \mathrm{~b}$ & Muralla & $3.03 \mathrm{~b}$ & Tracer & $0.33 \mathrm{~b}$ \\
\hline $\mathrm{T} 4$ & Tracer & $1.37 \mathrm{a}$ & Movento & $0.62 \mathrm{~b}$ & Curacron & $0.15 \mathrm{~b}$ & Muralla & $0.44 \mathrm{~b}$ \\
\hline $\mathrm{T} 5$ & Actigard & $3.13 \mathrm{a}$ & Tracer 100 & $0.45 \mathrm{~b}$ & Colt 350 & $8.68 \mathrm{~b}$ & Agromectin & $0.44 \mathrm{~b}$ \\
\hline \multicolumn{2}{|c|}{ Media general } & 3.01 & & 2.7 & & 15.25 & & 0.96 \\
\hline \multicolumn{2}{|c|}{ CV. $(\%)$} & 22 & & 29 & & 22 & & 21 \\
\hline
\end{tabular}

En columnas para cada factor letras similares indican que son estadísticamente iguales (Turkey, $\alpha=0.05$ ). 
Cuadro 3. Separación de medias de los tratamientos aplicados para el control de adultos de trips.

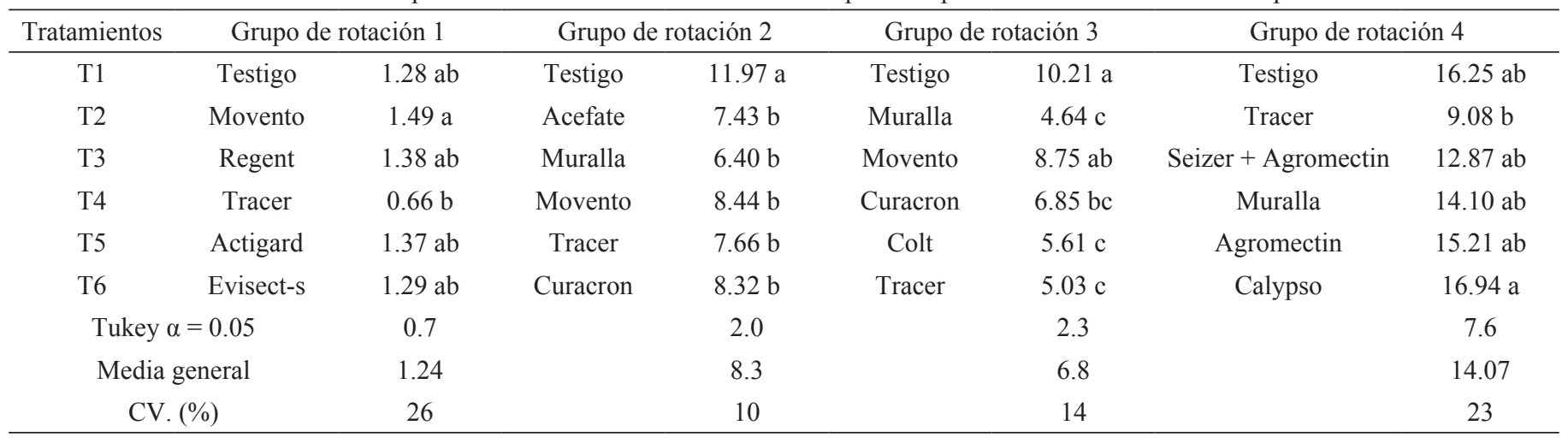

En columnas para cada factor letras similares indican que son estadísticamente iguales (Tukey, $\alpha=0.05$ ).

En el tercer grupo de rotación de insecticidas, Imidacloprid + Betacyflutrin y Propenofos fueron los que presentaron el menor número de ninfas con respecto al testigo y los otros insecticidas. Estos resultados fueron similares a los descritos por Cleveland et al. (2001) y Workman \& Martin (2002), quienes registraron el mejor control en ninfas con la aplicación de Spinosad e Imidacloprid en cultivo de cebolla. Con relación al cuarto grupo, el mejor control de ninfas de T. tabaci fue con Seizer + Agromectin, seguido de Spinosad; este resultado concuerda con lo encontrado por Workman \& Martin (2002), los cuales observaron el mayor control con Spinosad.

Efecto de los grupos de rotación de insecticidas 1, 2, 3 y 4 sobre la población de adultos de $T$. tabaci. Para las poblaciones de adultos, al aplicar el primer grupo de insecticidas, Tracer se comportó estadísticamente diferente al testigo, siendo el que presentó el menor número de adultos por planta. Dichos resultados se relacionan con los obtenidos por Valle de la Paz et al. (2003), donde Tracer fue uno de los productos más efectivos para el control de trips. También los resultados son similares a los obtenidos por Coria (2003), quien evaluó Tracer y obtuvo un buen control de trips. Al respecto, Salgado (1997) mencionó que una vez que el insecto ha ingerido o se pone en contacto con Spinosad la muerte puede ocurrir en un par de horas.

Con respecto al segundo y tercer grupo de insecticidas, Imidacloprid + Betacyflutrin mantuvo el promedio más bajo de la población de adultos. Resultados similares obtuvieron Al Mazraáwi (2007) y Workman \& Martin (2002), en el control de T. tabaci con la aplicación de imidacloprid en cebolla.

Para el ultimó muestreo, el número de adultos se incrementó en todos los tratamientos con relación al mues- treo anterior, esto debido a que las plantas de la unidad experimental donde se encontraba el testigo estaba en mal estado, por lo que se supone que el adulto de trips prefirió emigrar a plantas de mejor calidad. Sin embargo, en el cuarto grupo de insecticidas aplicados, el mejor control de adultos fue con Tracer, respecto al testigo, debido a que presentó el menor número de adultos (Cuadro 3); Workman \& Martin (2002) obtuvieron resultados similares.

\section{CONCLUSIÓN}

Los insecticidas que logran un control de las ninfas de trips en el cultivo de cebolla, fueron el Tracer, Acefate, Curacron y la combinación de Seizer + Agromectin; en tanto que los insecticidas Tracer y Muralla, fueron los únicos que mantuvieron las poblaciones más bajas de adultos de trips por planta en el cultivo de cebolla. La especie de trips predominante en el cultivo de cebolla durante el estudio fue Thrips tabaci.

\section{LITERATURA CITADA}

Al Mazraáwi, M. S. (2007) Interaction effects between Beauveria bassiana and imidacloprid against Thrips tabaci (Thysanoptera: Thripidae). Communications in Agricultural and Applied Biological Sciences, 72, 549-555.

Alston, D. G. (2008) Onion thrips (Thrips tabaci). Utah Pests Fact Sheet. ENT-117-08PR. Utah State University, 7 pp.

Burnstone, J. A. (2009) Investigations into the biology and behaviour of Thrips tabaci L. University of Warwick. PhD Thesis, 178 pp.

Cleveland, B., Mayes, A. \& Cryer, A. (2001) An ecological risk assessment for spinosad use on cotton. Pest Management Science, $58,70-84$. 
Coria, A. V. M. (2003) Fluctuación poblacional de trips y efectividad de un insecticida biológico para su control en aguacate. Agricultura Técnica en México, 29, 193-200.

FAO (2010) Agriculture Production and Trade Statistics. Food and Agriculture Organization of the United Nations, Rome. Available at: http://faostat.fao.org (accessed on November 2013).

García, M. M. (2003) Plagas, enfermedades y fisiopatías del cultivo de la Cebolla en la Comunidad Valenciana. Ed. Generalitat Valenciana Consellería de Agricultura, Pesca y Alimentación, 109 pp.

García-Gutiérrez, C. \& González-Maldonado, M. B. (2010) Uso de bioinsecticidas para el control de plagas de hortalizas en comunidades rurales. Ra Ximhai, 6, 17-22.

Johansen, R. M. \& Mojica, G. A. (1997) Importancia agrícola de los Thrips, pp. 11-18. In: Manual sobre entomología y acarología aplicada. UPAEP, Puebla, Pue. SME-UPAEP.

Lewis, T. (1973) Thrips: their biology, ecology and economic importance. London, Academic Press, $452 \mathrm{pp}$.

Liñán, V. C. \& Liñan, C. C. (2012) Texto Agroquímicos de México. Cuarta edición. Editorial TecnoAgrícola de México, 94 pp.

Riley, D. G., Joseph, S. V., Srinivasan, R. \& Diffie, S. (2011) Thrips vectors of tospoviruses. Journal of Integrated Pest Management, 2, 1-10.

Mound, L. A. \& Kibby, G. (1998) Thysanoptera an Identification Guide. Second Edition. CAB International, $67 \mathrm{pp}$.

Mound, L. A. \& Marullo, R. (1996) The Thrips of Central and South America: An Introduction (Insecta: Thysanoptera). Memory on Entomology International, 6, 1-487.

Osuna-Canizalez, F. J. \& Ramírez-Rojas, S. (2013) Manual para cultivar cebolla con fertirriego y riego por gravedad en el estado de Morelos. SAGARPA. INIFAP. CIRPAS. Campo Experimental Zacatepec. Libro Técnico No. 12, 155 pp.

Palmer, J. M., Mound, L. A. \& Heaume, G. J. (1989) CIE Guide to insects of importance to man. 2. Thysanoptera. CAB International, Wallngford; UK, $73 \mathrm{pp}$.
Ramírez, R. S., Osuna, C., F. de J., Guemes, G., M. J., Bartolo, R., J. C., Ocampo, O., T. \& Ayala, S. A. (2010) Plagas y enfermedades de la cebolla. Primera edición. INIFAP. Zacatepec, Morelos, $29 \mathrm{p}$.

Reveles-Hernández, M., Velásquez-Valle, R., Reveles-Torres L. R. \& Cid-Ríos J. A. (2014) Guía para producción de cebolla en Zacatecas. Folleto Técnico No. 62 Campo Experimental Zacatecas, CIRNOCINIFAP. Calera, Zacatecas, México, 40 pp.

SAS Institute (2003) SAS/STAT user's guide Release 9.1. SAS Institute. Cary, NC, USA.

Sakimura, K. (1937) The life and seasonal histories of Thrips tabaci Lind. in the vicinity of Tokyo, Japan. Oyo Dubues, Zasshi, 9, 124.

Salgado, L. (1997) The modes of action of spinosad and other insect control products. Down to Earth, 52, 35-43.

SIAP. (2013) Servicio de información Agroalimentaria y Pesquera. Cierre de producción Anual por cultivo 2012. Disponible en http:// reportes.siap.gob.mx/Agricola_siap/ResumenProducto.do ?producto $=7400 \&$ invitado $=$ true $\&$ ciclo $)=1$. (consultado en septiembre de 2013).

Turini, T. (2011) Thrips control programs y population dynamics in central SJV. [on line]. http://cetulare.ucanr.edu/files/137892.pdf (Consultado en septiembre de 2013).

Valle-De la Paz, M., Solís-Aguilar, J. F., Morales-García, J. L. \& Johansen-Naime, R. M. (2003) Efectividad biológica de productos no convencionales contra trips en el cultivo de aguacate (Persea americana mil. cv. has) en nuevo San Juan Parangaricutiro, Michoacán, México. V World Avocado Congress, pp. 735-740.

Velásquez, V. R. \& Reveles, H. M. (2011) Detección del iris yellow spot virus en el cultivo de cebolla en Zacatecas, México. Revista Mexicana de Ciencias Agrícolas, 2, 71-97.

Workman, P. J. \& Martin N. A. (2002) Towards integrated pest management of Thrips tabaci in onions. New Zealand Plant Protection, 55, 188-192. 